\title{
Intermittent fasting increases SOD2 and catalase immunoreactivities in the hippocampus but does not protect from neuronal death following transient ischemia in gerbils
}

\author{
JI HYEON AHN ${ }^{1 *}$, YOOHUN NOH $^{2 *}$, BICH NA SHIN $^{3}$, SUNG-SU KIM $^{2}$, JOON HA PARK $^{1}$, \\ TAE-KYEONG LEE ${ }^{4}$, MINAH SONG ${ }^{4}$, HYUNJUNG KIM $^{4}$, JAE-CHUL LEE ${ }^{4}$, JUN-HWAN YONG $^{5}$, \\ IL JUN KANG ${ }^{6}$, YUN LYUL LEE ${ }^{7}$, MOO-HO WON ${ }^{4}$ and JONG DAI KIM ${ }^{8}$
}

\author{
${ }^{1}$ Department of Biomedical Science and Research Institute for Bioscience and Biotechnology, Hallym University, Chuncheon, \\ Gangwon 24252; ${ }^{2}$ Famenity Company, Gwacheon, Geyonggi 13837; ${ }^{3}$ Danchunok Company, Chuncheon, Gangwon 24210; \\ ${ }^{4}$ Department of Neurobiology, School of Medicine, Kangwon National University, Chuncheon, Gangwon 24341; \\ ${ }^{5}$ Department of Occupational Therapy, Dongnam Health University, Suwon, Gyeonggi 16238; \\ ${ }^{6}$ Department of Food Science and Nutrition, Hallym University, Chuncheon, Gangwon 24252; ${ }^{7}$ Department of Physiology \\ and Institute of Neurodegeneration and Neuroregeneration, College of Medicine, Hallym University, \\ Chuncheon, Gangwon 24252; ${ }^{8}$ Division of Food Biotechnology, School of Biotechnology, \\ Kangwon National University, Chuncheon, Gangwon 24341, Republic of Korea
}

Received April 23, 2018; Accepted September 11, 2018

DOI: $10.3892 / \mathrm{mmr} .2018 .9503$

\begin{abstract}
Intermittent fasting has been shown to have neuroprotective effects against transient focal cerebral ischemic insults. However, the effects of intermittent fasting on transient global ischemic insult has not been studied much yet. The present study examined effects of intermittent fasting on endogenous antioxidant enzyme expression levels in the hippocampus and investigated whether the fasting protects neurons 5 days after $5 \mathrm{~min}$ of transient global cerebral ischemia. Gerbils were randomly subjected to either ad libitum or alternate-day intermittent fasting for two months and assigned to sham surgery or transient ischemia. Changes of antioxidant enzymes were examined using immunohistochemistry for cytoplasmic superoxide dismutase 1 (SOD1), mitochondrial (SOD2), catalase (CAT), and glutathione peroxidase (GPX). The effects of intermittent
\end{abstract}

Correspondence to: Professor Moo-Ho Won, Department of Neurobiology, School of Medicine, Kangwon National University, 1 Gangwondaehak, Chuncheon, Gangwon 24341, Republic of Korea E-mail:mhwon@kangwon.ac.kr

Professor Jong Dai Kim, Division of Food Biotechnology, School of Biotechnology, Kangwon National University, 1 Gangwondaehak, Chuncheon, Gangwon 24341, Republic of Korea

E-mail: jongdai@kangwon.ac.kr

*Contributed equally

Key words: antioxidants, astrocyte, hippocampus, intermittent fasting, ischemia-reperfusion, microglia fasting on ischemia-induced antioxidant changes, neuronal damage/degeneration and glial activation were examined. The weight of fasting gerbils was not different from that of control gerbils. In controls, SOD1 and GPX immunoreactivities were strong in pyramidal neurons of filed cornu ammonis 1 (CA1). Transient ischemia in controls significantly decreased expressions of SOD1 and GPX in CA1 pyramidal neurons. Intermittent fasting resulted in increased expressions of SOD2 and CAT, not of SOD1 and GPX, in CA1 pyramidal neurons. Nevertheless, CA1 pyramidal neurons were not protected in gerbils subjected to fasting after transient ischemia, and inhibition of glial-cell activation was not observed in the gerbils. In summary, intermittent fasting for two months increased SOD2 and CAT immunoreactivities in hippocampal CA1 pyramidal neurons. However, fasting did not protect the CA1 pyramidal neurons from transient cerebral ischemia. The results of the present study indicate that intermittent fasting may increase certain antioxidants, but not protect neurons from transient global ischemic insult.

\section{Introduction}

Pyramidal neurons in the hippocampal cornu ammonis 1 (CA1) area are killed four to five days after brief ( 5 to $10 \mathrm{~min}$ ) transient global cerebral ischemia in gerbils (1). A complex series of molecular mechanisms of ischemia-induced neuronal degeneration/death is related to increased glutamate excitotoxicity, oxidative stress, and inflammation (2,3). Many researchers have been struggling to find relevant molecular targets from those mechanisms to protect neurons against ischemic damage for developing therapeutics. For example, protective effects of antioxidants, superoxide dismutases (SODs), and their mimetics, have been demonstrated $(4,5)$. 
Intermittent fasting (IF) is defined as a severe dietary energy restriction during a certain period of normal energy intake (6). The duration of IF is variable, and previous studies have typically used alternate-day fasting or a daily time-restricted (4 to1 $2 \mathrm{~h}$ ) food-deprivation regimen in rodents (rats or mice) (7). It has been reported that the liver and adipose tissue act as energy storage, allowing fasting for various periods in mammals (8). Additionally, during fasting, body systems, including the metabolic, endocrine, and nervous systems, adjust to enable a high level of physical and mental activities (8).

Beneficial effects of dietary restriction are increased resistance to aging and degenerative diseases, and their associated mechanisms have been demonstrated in previous studies. IF activates the sirtuin 1 signaling pathway, which plays a major role in life span and cellular health, and decreases apoptotic pathways in the brain of senescence-accelerated mice p8 (SAMP8) (9). In addition, IF-related insulin-like signaling and FoxO transcription factors are known to stimulate antioxidant enzymes to help cells resist stress (10).

For the brain, many researchers have studied whether an IF regimen provides neuroprotection or not. Zhu et al (11), have reporetd that IF protects hippocampal neurons against kainate excitotoxicity in a mouse model of Alzheimer's disease (presenilin1 mutant knockin mice) by reducing oxidative stress. In ischemia, it has been demonstrated that dietary restriction or an IF regimen reduces infarct volume in rodent models of cerebral focal cerebral ischemia by inhibiting the accumulation of autophagosomes in neurons (12), by suppressing inflammasome activity (13), and by increasing a preconditioning stress response (14). The above-mentioned studies attenuate or protect ischemic damage in focal cerebral ischemia models; however, the possibility that IF protects neurons from transient global cerebral ischemia (tGCI) has not been examined. Therefore, in this study, we investigated effects of IF on expressions of endogenous antioxidant enzymes, and then examined the effect of IF on expressions of antioxidant enzymes, neuronal damage/degeneration, and reactive glia cells following tGCI in gerbils, which are a good animal model of tGCI $(15,16)$.

\section{Materials and methods}

Experimental animals. Male gerbils were obtained at 6 months of age (B.W., 70 $\pm 5.2 \mathrm{~g}$ ) from the Experimental Animal Center, Kangwon University, Chuncheon, Gangwon, Republic of Korea, and maintained at a constant temperature $\left(23^{\circ} \mathrm{C}\right)$ and humidity $(50 \%)$ with a 12 -h light/dark cycle. The process of handling and caring animals conformed to the guidelines being in compliance with current international laws and policies (NIH Guide for the Care and Use of Laboratory Animals, The National Academies Press, 8th ed., 2011). The protocol of this experiment was approved by the Institutional Animal Care and Use Committee (IACUC) at Kangwon National University (approval no. KW-180124-1).

IF and experimental groups. Animals were fed commercially available rodent normal diet or IF ( $24 \mathrm{~h}$ fasting and $24 \mathrm{~h}$ feeding) was applied for 2 months according to method by published methods $(9,12,17)$. During procedures, food intake of IF group was controlled daily (10 g per day), and body weight of normal diet and IF groups was monitored every week. After 2 months, animals with normal diet or IF were randomly assigned to following groups: i) Sham groups $(n=7)$, which were allowed free access to water and food and received no ischemia; ii) IF and sham (IF+Sham) group $(n=7)$, which was subjected to IF and received no ischemia; iii) Ischemia groups $(n=7)$, which received tGCI without IF and iv) IF+Ischemia groups $(n=7)$, which were subjected to IF and received tGCI. To investigate effects of IF on neuronal death (loss), antioxidant enzymes, and gliosis, all animals were sacrificed at 5 days after ischemia, because death (loss) of pyramidal neurons in the gerbil hippocampal CA1 region occurs 5 days follwong transient cerebral ischemia (1).

Induction of $t G C I$. As previously described (18), in brief, gerbils in all groups were anesthetized with a mixture of $2.5 \%$ isoflurane (Baxtor, Deerfield, IL, USA) in 33\% oxygen and $67 \%$ nitrous oxide. The gerbils received a midline incision on the ventral surface of the neck, and both common carotid arteries were occluded for 5 min using non-traumatic aneurysm clips. We controlled normal body (rectal) temperature $\left(37 \pm 0.5^{\circ} \mathrm{C}\right)$ using a thermometric blanket throughout the surgery, monitoring the temperature with a rectal temperature probe (TR-100; Fine Science Tools, Foster City, Inc., CA, USA).

Preparation of histological sections. For histology, as described previously (18), gerbils were anesthetized with $30 \mathrm{mg} / \mathrm{kg}$ Zoletil 50 (Virbac, Carros, France) 5 days after tGCI (at this point in time, pyramidal neurons are dead after tGCI), and perfused transcardially with $0.1 \mathrm{~m}$ phosphate buffered saline (PBS, pH 7.4) followed by $4 \%$ paraformaldehyde in $0.1 \mathrm{~m}$ phosphate buffer (PB, $\mathrm{pH}$ 7.4). The brain tissues containing hippocampi were cryoprotected and serially sectioned into $30-\mu \mathrm{m}$ coronal sections in a cryostat (Leica Microsystems GmbH, Wetzlar, Germany).

Immunohistochemistry. In brief, according to our published method (19), sheep anti-superoxide dismutase 1 (SOD1) (1:1,000; EMD Millipore, Billerica, MA, USA), sheep anti-mitochondrial (SOD2) (1:1,000; EMD Millipore), rabbit anti-catalase (CAT) (1:500; EMD Millipore), mouse anti-glutathione peroxidase (GPX) (1:500; EMD Millipore), mouse anti-NeuN (a marker for neuron) (1:1,000; Thermo Fisher Scientific, Inc., Waltham, MA, USA), mouse anti-GFAP (a marker for astrocyte) (1:800; Abcam, Cambridge, MA, USA), and rabbit anti-Ibal (a marker for microglia) (1:800; Wako Pure Chemical Industries, Ltd., Osaka, Japan) were used as primary antibodies. The sections were sequentially treated with $0.3 \%$ hydrogen peroxide $\left(\mathrm{H}_{2} \mathrm{O}_{2}\right)$ in PBS for $30 \mathrm{~min}$ and $10 \%$ normal goat serum in $0.05 \mathrm{M}$ PBS for $30 \mathrm{~min}$. The treated sections were incubated with the primary antibodies overnight at $4^{\circ} \mathrm{C}$, thereafter, the reacted sections were exposed to biotinylated goat anti-mouse or goat anti-rabbit IgG (1:200; Vector Laboratories, Inc., Burlingame, CA, US) and streptavidin peroxidase complex (1:200; Vector Laboratories, Inc.). Finally, the reacted sections were visualized by staining with 3, 3'-diaminobenzidine tetrahydrochloride in $0.1 \mathrm{M}$ Tris- $\mathrm{HCl}$ buffer ( $\mathrm{pH}$ 7.2).

Fluoro-Jade (F-J) B histofluorescence staining. To investigate neuronal death in the hippocampal CA1 at 5 days after tGCI, F-J B (a fluorescent marker for cell degeneration) histofluorescence staining was conducted according to method published 
by Candelario-Jalil et al (20). In brief, the sections were first immersed in a solution containing $1 \%$ sodium hydroxide in $80 \%$ alcohol and followed in $70 \%$ alcohol. They were then transferred to a solution of $0.06 \%$ potassium permanganate, and to a $0.0004 \%$ F-J B (Histochem, Inc., Jefferson, AR, USA) staining solution. After washing them, they were placed on a slide warmer (approximately $50^{\circ} \mathrm{C}$ ) to be reacted. The stained sections were examined using an epifluorescent microscope (Carl Zeiss AG, Oberkochen, Germany) with blue (450-490 nm) excitation light and a barrier filter (Schmued and Hopkins, 2000).

Data analysis. First, we quantitatively analyzed SOD1, SOD2, GPX, CAT, GFAP, and Iba-1 immunoreactivities according to our published method (19). In brief, we selected six sections from each animal with $120-\mu \mathrm{m}$ interval according to AP (Antero-posterior) -1.4 to $-2.2 \mathrm{~mm}$ of the gerbil brain atlas and took images of them from the CA1 through an AxioM1 light microscope (Carl Zeiss AG) equipped with a digital camera (Axiocam; Carl Zeiss AG) connected to a PC monitor. The image of each immunoreactivity was calibrated into an array of $512 \times 512$ pixels corresponding to a tissue area of $250 \times 250 \mu \mathrm{m}^{2}$ (20x primary magnification). Each immunoreactivity was measured by a 0-255 gray scale system and evaluated by optical density (OD), which was obtained after transformation of the mean gray level using the formula: $\mathrm{OD}=\log (255 /$ mean gray level). A ratio of the OD was calibrated as \% (relative OD, ROD) using Adobe Photoshop version 8.0 and analyzed using Image J 1.46 software (National Institutes of Health, Bethesda, MD, USA). A ratio of the ROD was calibrated as $\%$, with the Sham group designated as $100 \%$.

Second, we analyzed numbers of NeuN- and F-J B-positive cells according to our published method (19). In brief, we selected six sections like the above-mentioned method. Images of NeuN- and F-J B-positive cells were captured through an AxioM1 light microscope (Carl Zeiss AG) equipped with a digital camera (Axiocam; Carl Zeiss AG) connected to a PC monitor. CA1 pyramidal neurons were captured in a $250 \times 250 \mu \mathrm{m}$ square. Cell counts were obtained by averaging the total number of NeuN- and F-J B-positive cells from each animal using an image analyzing system (Optimas v.6.5; CyberMetrics, Scottsdale, AZ, USA).

Statistical analysis. The data shown here represent the means \pm SEM. Differences of the means among the groups were statistically analyzed by one-way analysis of variance with Duncan's post hoc test using SPSS v.17.0 software (SPSS, Inc., Chicago, IL, USA). $\mathrm{P}<0.05$ was considered to indicate a statistically significant difference.

\section{Results}

Body weight. Normal diet or IF was treated for 2 months. Body weight in the normal diet animals was slowly increased. Change in body weight in the IF animals was not significantly different from that in the normal diet animals (Fig. 1).

\section{Immunoreactivities of antioxidant enzymes}

SOD1 immunoreactivity. When we examined SOD1 immunoreactivity in the Sham group, SOD1 immunoreactivity was mainly shown in neurons of the stratum pyramidale in the CA1, which are called CA1 pyramidal neurons (Fig. 2A). In the IF+Sham

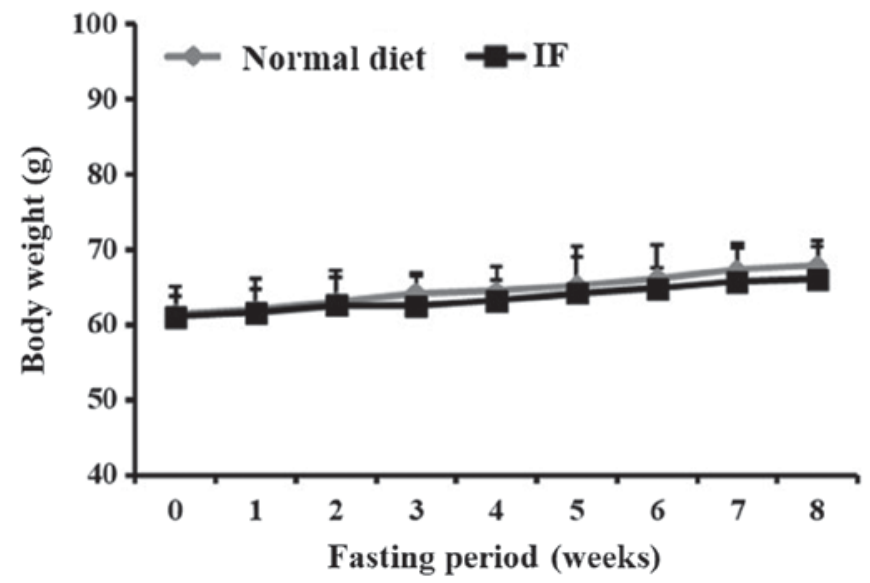

Figure 1. Change in body weight in animals with normal diet or IF for two months. Weight gain in the animals with normal diet and IF is not significantly different between them ( $\mathrm{n}=14$ at each point in time). Bars indicate the means \pm SEM

group, SOD1 immunoreactivity in CA1 pyramidal neurons was not different from that in the Sham group (Fig. 2B).

In the Ischemia group, SOD1 immunoreactivity was hardly found in CA1 pyramidal neurons, but increased in many non-pyramidal cells in stratum oriens and radiatum of the CA1, and the SOD1 immunoreactivity was increased by about $43 \%$ compared to the Sham group (Fig. 2C). In the IF+Ischemia group, the pattern and immunoreactivity of SOD1 in the CA1 was similar to that in the Ischemia group (Fig. 2D).

Each immunoreactivity of SOD1 in the CA1 region at 5 days after tGCI in the Sham, IF+Sham, Ischemia, and $\mathrm{IF}+\mathrm{Ischemia}$ groups was shown in Fig. 2E.

SOD2 immunoreactivity. In the Sham group, very weak SOD2 immunoreactivity was detected in CA1 pyramidal neurons (Fig. 3A). In the IF+Sham group, SOD2 immunoreactivity in CA1 pyramidal neurons was significantly increased by about $40 \%$ compared to the Sham group (Fig. 3B).

In the Ischemia group, SOD2 immunoreactivity was rarely shown in CA1 pyramidal neurons, instead, SOD2 immunoreactivity was increased in non-pyramidal cells (Fig. 3C). In the IF+Ischemia group, the pattern of SOD2 expression in the CA1 was not different from the Ischemia group; however, SOD2 immunoreactivity in non-pyramidal cells was increased by about $41 \%$ compared to the Ischemia group (Fig. 3D).

Each immunoreactivity of SOD2 in the CA1 region at 5 days after tGCI in the Sham, IF+Sham, Ischemia, and $\mathrm{IF}+\mathrm{Ischemia}$ groups was shown in Fig. 3E.

CAT immunoreactivity. CAT immunoreactivity was observed in CA1 pyramidal neurons in the Sham group (Fig. 4A). In the IF+Sham group, CAT immunoreactivity in CA1 pyramidal neurons was increased by about $102 \%$ compared to the Sham group (Fig. 4B).

In the Ischemia group, CAT immunoreactivity was hardly found in CA1 pyramidal neurons, instead, weak CAT immunoreactivity was shown in non-pyramidal cells (Fig. 4C). In the IF+Ischemia group, CAT immunoreactivity was strong in many non-pyramidal cells, and the immunoreactivity was increased by about $85 \%$ compared to the Ischemia group (Fig. 4D). 
$\mathbf{A}$

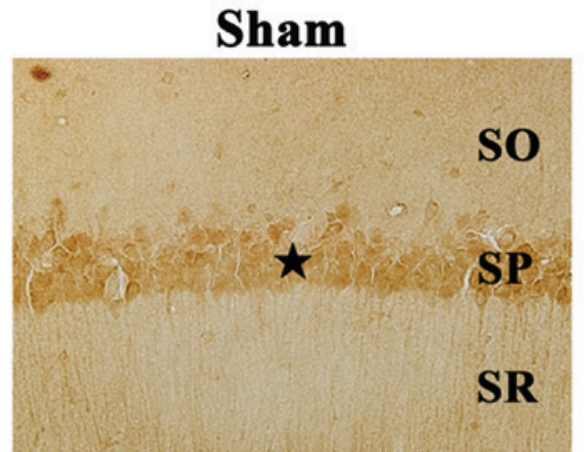

C

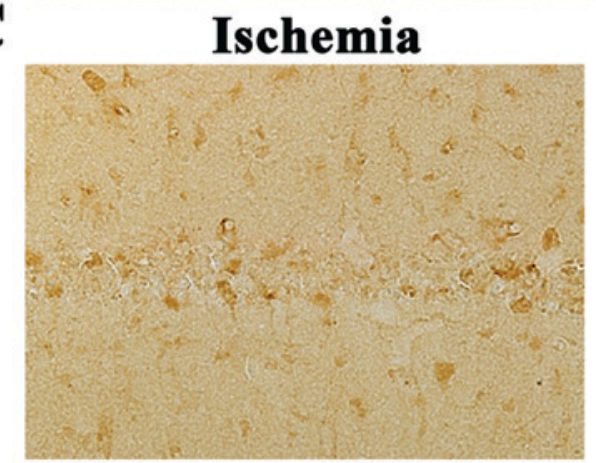

B

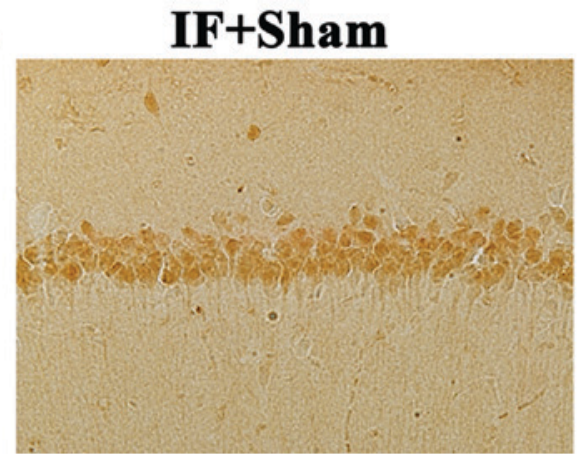

D

IF+Ischemia

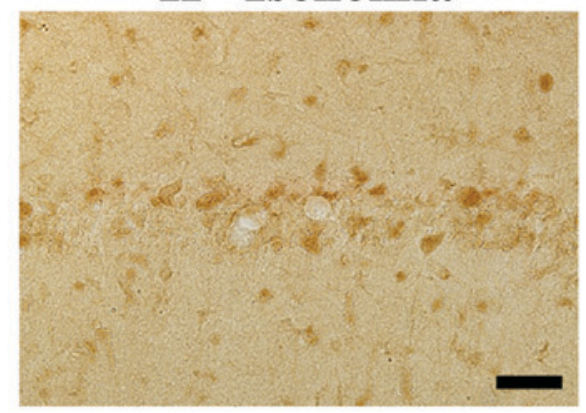

$\mathbf{E}$

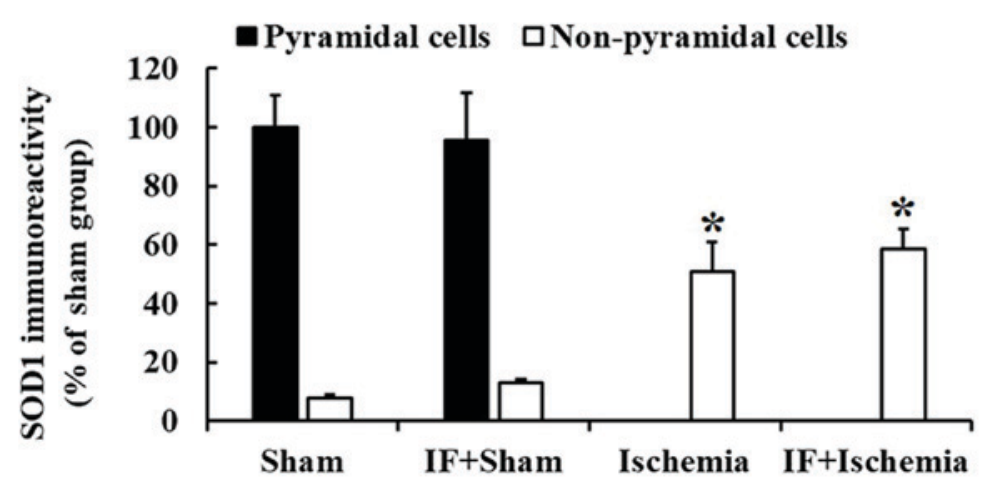

Figure 2. SOD1 immunohistochemistry. Immunoreactivities of SOD1 in the CA1 of (A) Sham, (B) IF+Sham, (C) Ischemia, and (D) IF+Ischemia groups 5 days after tGCI. In the Sham group, SOD1 immunoreactivity is shown in pyramidal neurons (star in A). SOD1 immunoreactivity in the IF+Sham group is similar to the Sham group. In the Ischemia group, SOD1 immunoreactivity is found in non-pyramidal cells. SOD1 immunoreactivity in the IF+Sham group is not different from the Sham group. Scale bar=50 $\mu \mathrm{m}$. (E) SOD1 immunoreactivity as percent values in pyramidal and non-pyramidal cells ( $\mathrm{n}=7$ in each group, ${ }^{*} \mathrm{P}<0.05$ vs. Sham group). Bars indicate the means \pm SEM. SO, stratum oriens; SP, stratum pyramidale; SR, stratum radiatum.

Each immunoreactivity of CAT in the CA1 region at 5 days after tGCI in the Sham, IF+Sham, Ischemia, and IF+Ischemia groups was shown in Fig. 4E.

GPX immunoreactivity. Strong GPX immunoreactivity was found in CA1 pyramidal neurons in the Sham group (Fig. 5A). In the IF+Sham group, no significant difference in GPX immunoreactivity in CA1 pyramidal neurons was observed compared to the Sham group (Fig. 5B).

In the Ischemia group, GPX immunoreactivity in CA1 pyramidal neurons was hardly identified, instead, strong GPX immunoreactivity was observed in many non-pyramidal cells (Fig. 5C). In the IF+Ischemia group, GPX immunoreactivity in non-pyramidal cells was not different from that in the Ischemia group (Fig. 5D).

Each immunoreactivity of GPX in the CA1 region at 5 days after tGCI in the Sham, IF+Sham, Ischemia, and IF+Ischemia groups was shown in Fig. 5E.

\section{Neuroprotection}

NeuN-immunoreactive neurons. In the Sham group, NeuN-immunoreactive neurons, as pyramidal neurons, were predominantly distributed in the stratum pyramidale (Fig. 6A). In the IF+Sham group, NeuN-immunoreactive pyramidal neurons were not different in their distribution compared with the Sham group (Fig. 6B).

In the Ischemia group, numbers of NeuN-immunoreactive neurons was significantly decreased only in the stratum pyramidale of the CA1 (Fig. 6C). In the IF+Ischemia group, the distribution and numbers of NeuN-immunoreactive neurons were similar to the Ischemia group (Fig. 6D and E).

F-J B-positive cells. F-J B-positive cells, which are dead/degenerated cells, were not detected in the stratum pyramidale of the CA1 in the Sham group (Fig. 7A). In the $\mathrm{IF}+$ Sham group, F-J B-positive cells were not shown like the Sham group (Fig. 7B). 


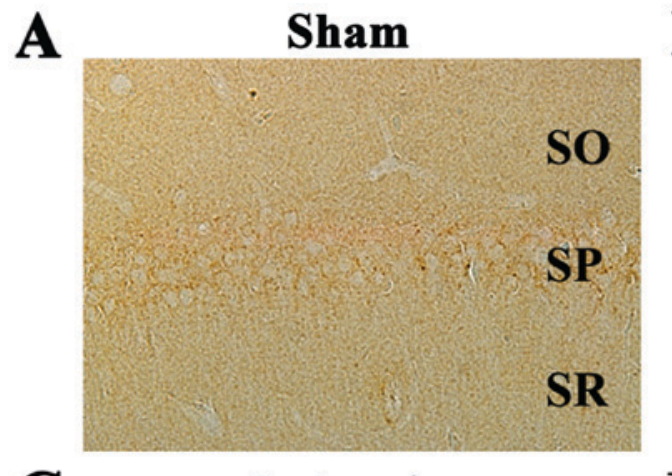

B

IF+Sham

C

Ischemia

D
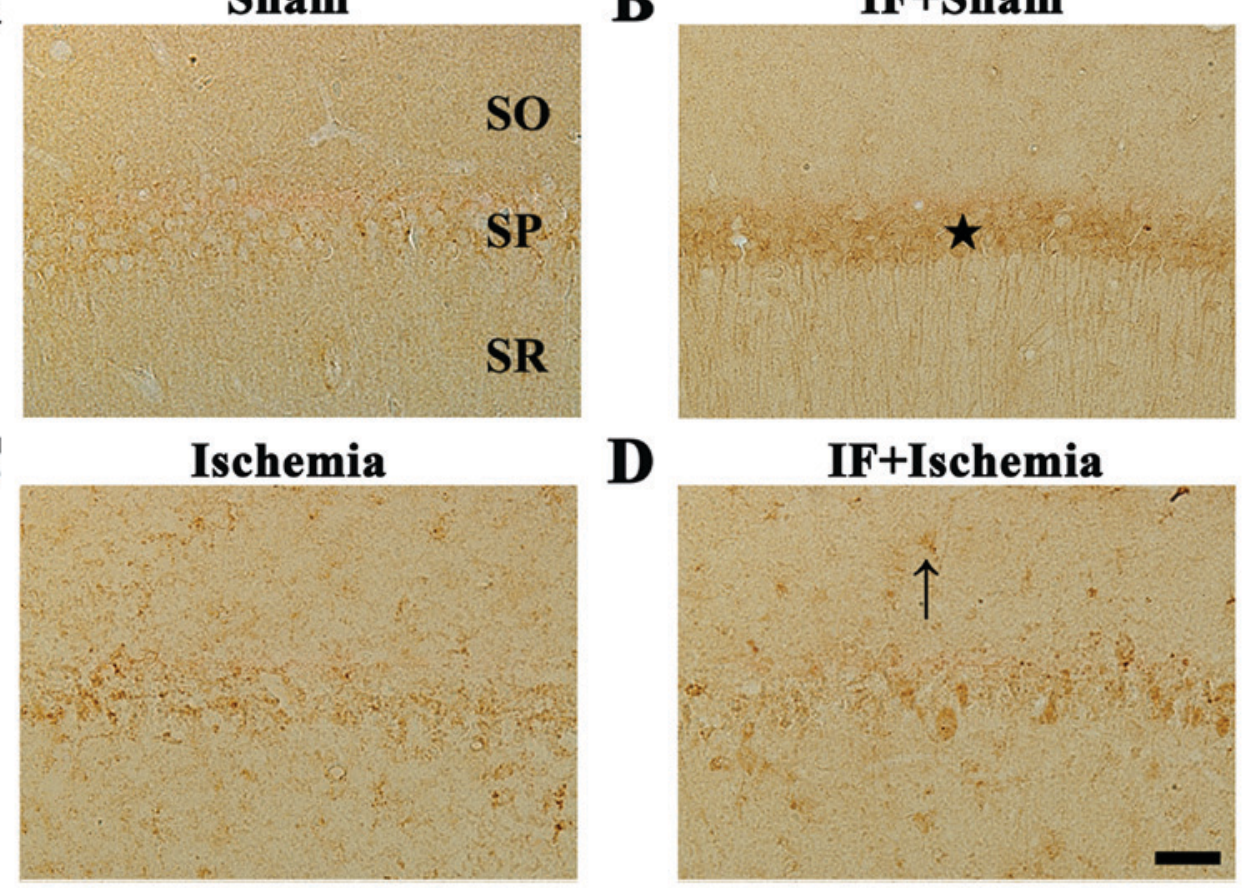

IF+Ischemia

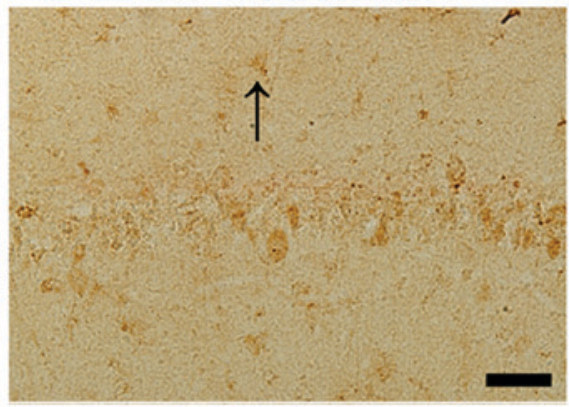

$\mathbf{E}$

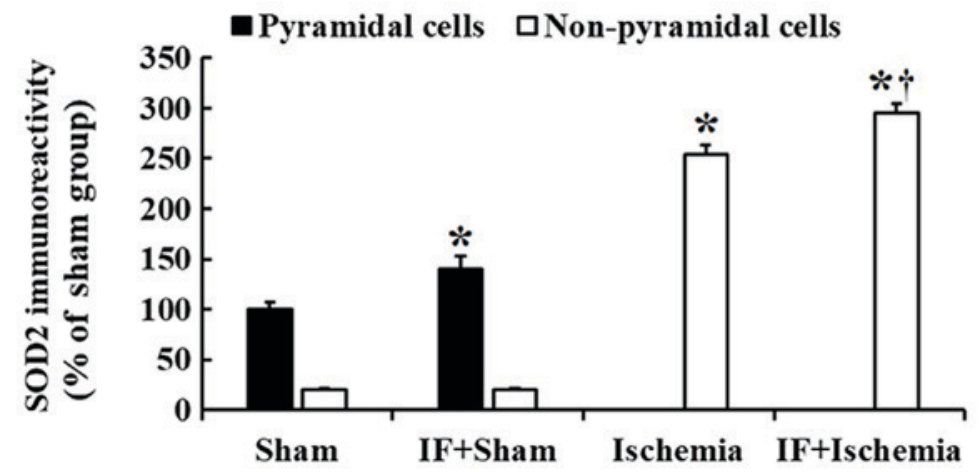

Figure 3. SOD2 immunohistochemistry. Immunoreactivities of SOD2 in the CA1 of (A) Sham, (B) IF+Sham, (C) Ischemia, and (D) IF+Ischemia groups 5 days after tGCI. SOD2 immunoreactivity is significantly increased in pyramidal neurons (star in B) compared to the Sham group. In the Ischemia group, SOD2 immunoreactivity is shown in non-pyramidal cells. SOD2 immunoreactivity in the IF+Ischemia is significantly increased (arrows in D) compared to the Ischemia group. Scale bar $=50 \mu \mathrm{m}$. (E) SOD2 immunoreactivity as percent values in pyramidal and non-pyramidal cells ( $\mathrm{n}=7$ in each group, ${ }^{*} \mathrm{P}<0.05 \mathrm{vs}$. Sham group, ${ }^{\dagger} \mathrm{P}<0.05$ vs. Ischemia group). Bars indicate the means \pm SEM. SO, stratum oriens; SP, stratum pyramidale; SR, stratum radiatum.

In the Ischemia group, many F-J B positive cells were shown in the stratum pyramidale of the CA1 (Fig. 7C). In the IF+Ischemia group, the distribution and numbers of F-J B-positive cells were similar to the Ischemia group (Fig. 7D and E).

\section{Glial activation and Inflammation}

GFAP immunoreactivity. In the Sham group, GFAP immunoreactive cells, which were astrocytes, had small cytoplasm and fine processes, and scattered throughout in all layers (Fig. 8A). In the IF+Sham group, the morphology and immunoreactivity of GFAP was similar to that in the Sham group (Fig. 8B).

In the Ischemia group, GFAP-immunoreactive cells displayed thick processes, and GFAP immunoreactivity was increased by about $279 \%$ compared to the Sham group (Fig. 8C). In the IF+Ischemia group, the morphology of GFAP-immunoreactive cells and GFAP immunoreactivity was similar to those in the Ischemia group (Fig. 8D).
Each immunoreactivity of GFAP in the CA1 region at 5 days after tGCI in the Sham, IF+Sham, Ischemia, and $\mathrm{IF}+\mathrm{Ischemia}$ groups was shown in Fig. 8E.

Iba-1 immunoreactivity. In the Sham group, Iba-1immunoreactive cells, which were microglia, had small cell body and distributed in all layers (Fig. 9A). In the $\mathrm{IF}+$ Sham group, the morphology and immunoreactivity of Iba-1-immunoreactive cells was not different from the Sham group (Fig. 9B).

In the Ischemia group, Iba-1-immunoreactive cells showed activated form with hypertrophied cell bodies and branched processes, and many of them were aggregated into and near the stratum pyramidale, showing that Iba-1 immunoreactivity was by about $226 \%$ compared to the Sham group (Fig. 9C). In the IF+Ischemia group, the distribution of Iba-1-immunoreactive cells and their Iba-1 immunoreactivity was not different from the Ischemia group (Fig. 9D). 

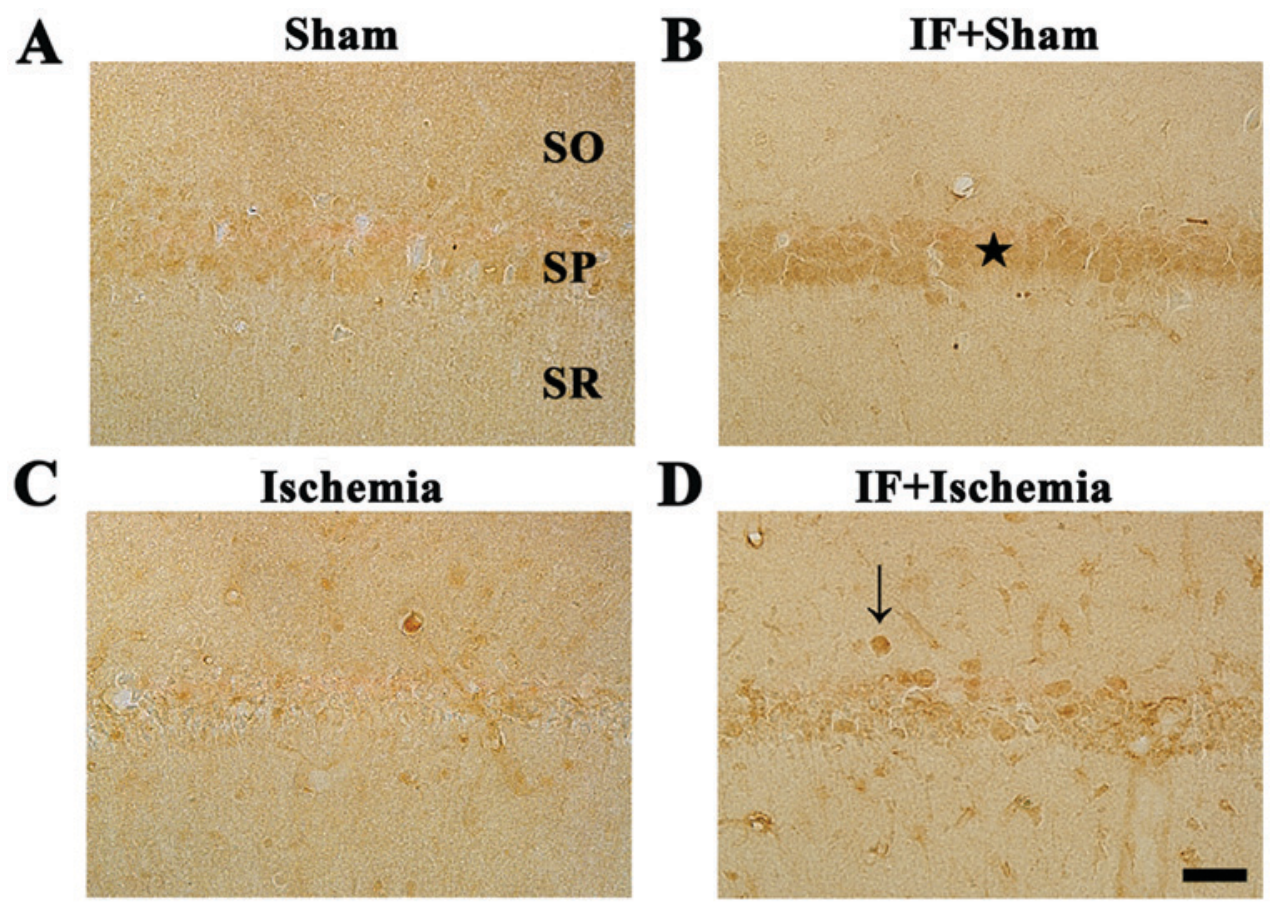

D
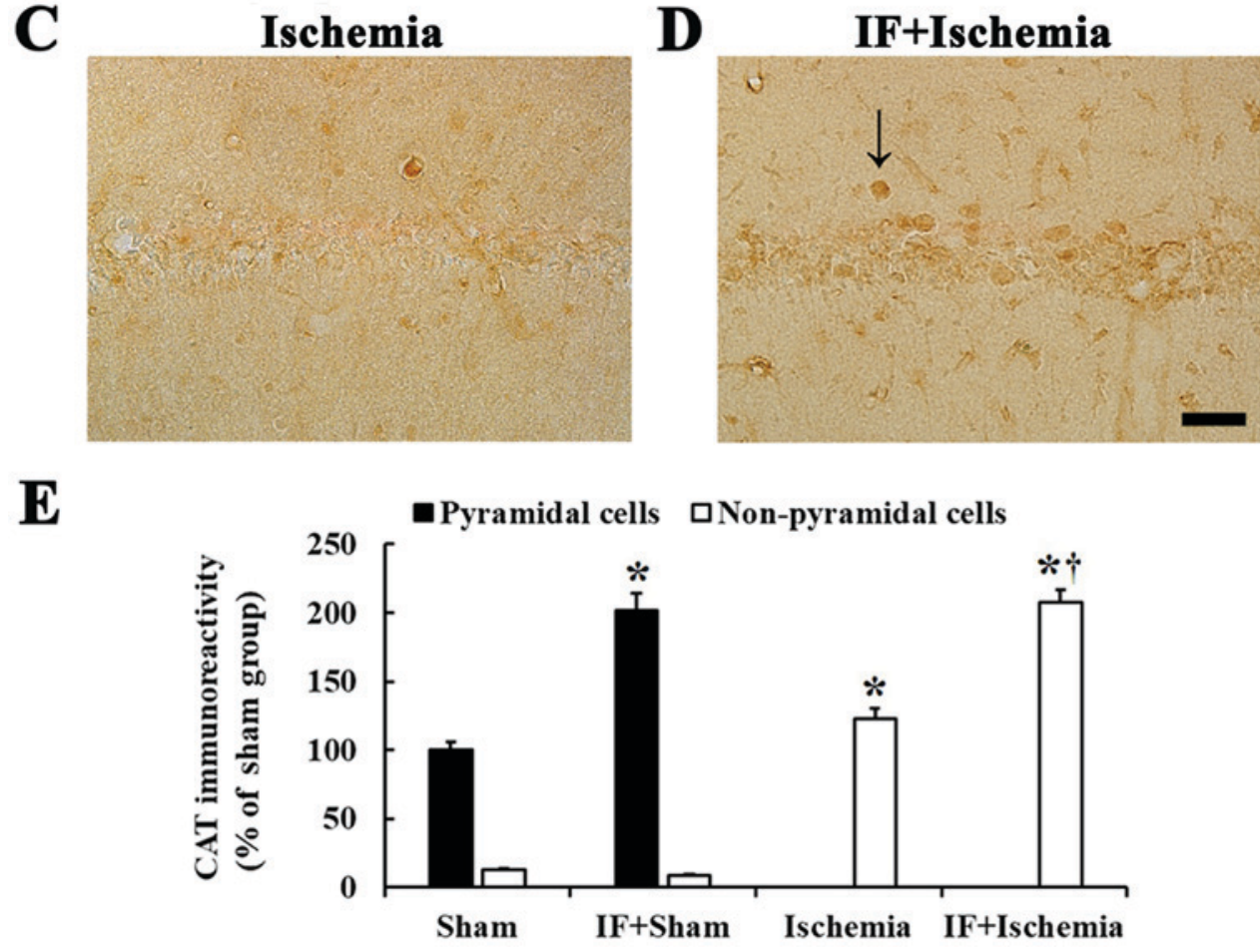

Figure 4. CAT immunohistochemistry. Immunoreactivities of CAT in the CA1 of (A) Sham, (B) IF+Sham, (C) Ischemia, and (D) IF+Ischemia groups 5 days after tGCI. In the IF+Sham group, CAT immunoreactivity is significantly increased in pyramidal neurons (star in B) compared to the Sham group. In the $\mathrm{IF}+\mathrm{Ischemia}$ group, CAT immunoreactivity is significantly increased in non-pyramidal cells (arrow in D) compared to the Ischemia group. Scale bar=50 $\mu \mathrm{m}$. SO, stratum oriens; SP, stratum pyramidale; SR, stratum radiatum. (E) CAT immunoreactivity as percent values in pyramidal and non-pyramidal cells (n=7 in each group, ${ }^{*} \mathrm{P}<0.05$ vs. Sham group, ${ }^{\top} \mathrm{P}<0.05$ vs. Ischemia group). Bars indicate the means $\pm \mathrm{SEM}$.

Each immunoreactivity of Iba-1 in the CA1 region at 5 days after tGCI in the Sham, IF+Sham, Ischemia, and IF+Ischemia groups was shown in Fig. 9E.

\section{Discussion}

This study was examined effects of IF on endogenous antioxidant enzymes, SOD1, SOD2, GPX and CAT, in the hippocampal CA1 of the gerbil, and investigated effects of IF on tGCI-induced antioxidant enzymes, neuronal damage/degeneration, and reactive glia cells.

We found that weight gain in the IF-sham group for two months was similar to that of the Sham group. In line with our results, it was reported that C57BL6 mice lost little or no weight, although other mice or rats lost weight after IF (21). These results indicate that weight loss after IF is different according to kinds or species of experimental animals. We need to study exact changes in weight gain or loss induced by IF in various kinds of experimental animals and its causes and mechanisms.
It is well known that SODs convert superoxide to hydrogen peroxide $\left(\mathrm{H}_{2} \mathrm{O}_{2}\right)$, and that $\mathrm{H}_{2} \mathrm{O}_{2}$ is converted to $\mathrm{H}_{2} \mathrm{O}$ by scavenger enzymes, such as CAT and GPX, to detoxify harmful radicals and reactive oxidative stress (22). It has been reported that SOD2 (mitochondrial enzyme) is a more important enzyme, since SOD2 knockout mice die earlier after birth or suffer from severe neurodegeneration (23), but SOD1 (cytosolic enzyme) knockout mice, which are phenotypically normal with only reproductive problems, can survive (24). Furthermore, it has been demonstrated that CAT removes peroxides more effectively than does GPX in neurons $(22,25)$. In a previous study, alternate-day fasting for four or five months has shown increased levels of heme oxygenase (HO)-1, an antioxidant enzyme in the mouse brain (26). Similar to the previous study, our current study showed that IF for two months significantly increased SOD2 and CAT immunoreactivities, but not SOD1 and GPX immunoreactivities in CA1 pyramidal neurons. These findings indicate that IF could induce increases of basal antioxidant expressions, especially SOD2 and CAT, in 

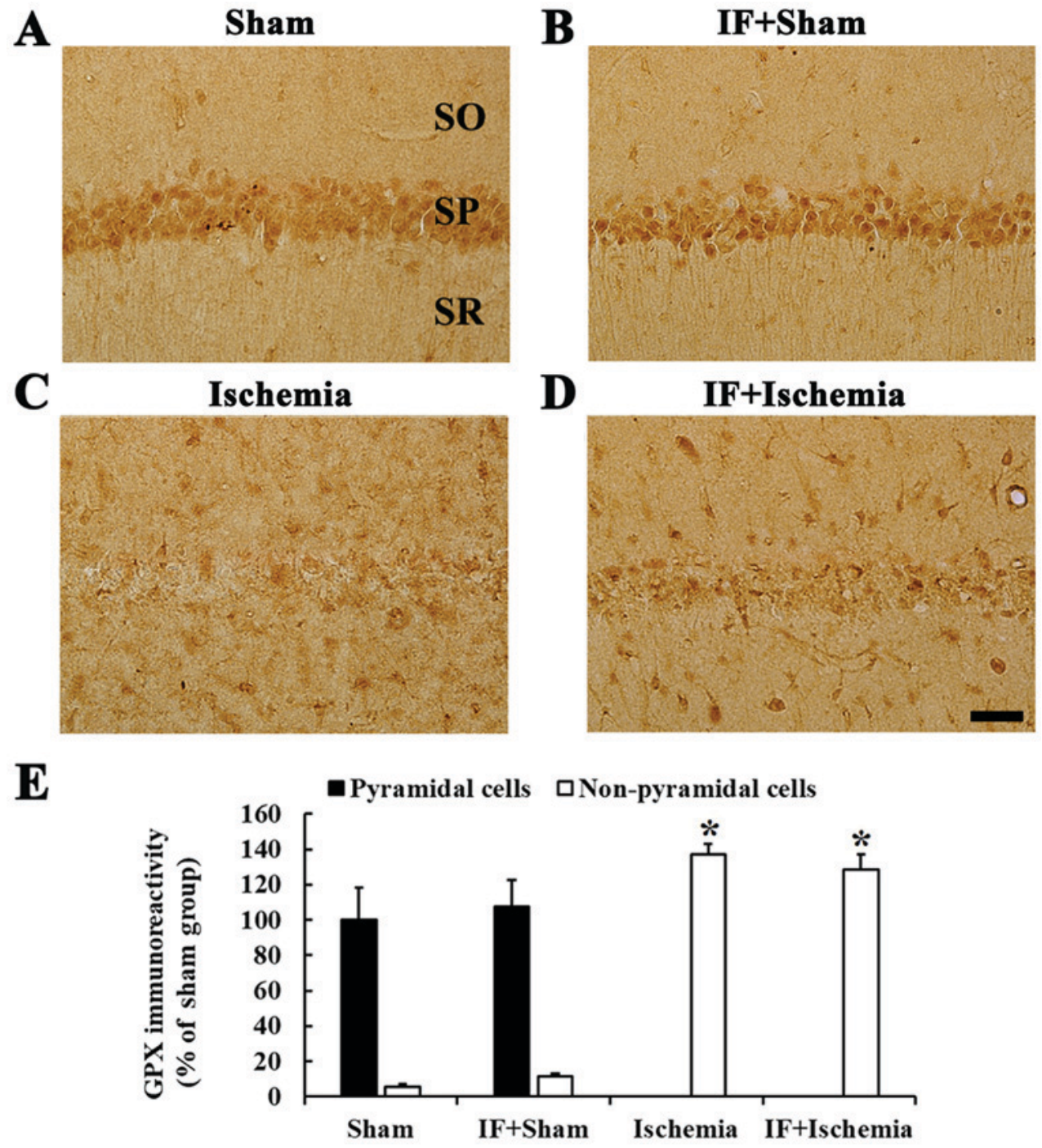

Figure 5. GPX immunohistochemistry. Immunoreactivities of GPX in the CA1 of (A) Sham, (B) IF+Sham, (C) Ischemia, and (D) IF+Ischemia groups 5 days after tGCI. GPX immunoreactivity in the IF+Sham group is shown in pyramidal neurons, and the immunoreactivity is not changed compared to the Sham group. In the IF+Ischemia, GPX immunoreactivity is shown in non-pyramidal cells, and the immunoreactivity is not different from the Ischemia group. Scale bar $=50 \mu \mathrm{m}$. SO, stratum oriens; SP, stratum pyramidale; SR, stratum radiatum. (E) GPX immunoreactivity as percent values in pyramidal and non-pyramidal cells ( $\mathrm{n}=7$ in each group, ${ }^{\mathrm{P}}<0.05$ vs. Sham group). Bars indicate the means \pm SEM.

hippocampal CA1 pyramidal neurons in gerbils, which have been used for an animal model of tGCI $(15,16)$.

We have demonstrated that neuronal protection or improvement of neuronal survival (after drug treatment) is closely related to maintenance or increase of SOD1, SOD2, CAT, and GPX expressions in the gerbil hippocampus after $5 \mathrm{~min}$ of tGCI $(19,27,28)$. In addition, Walsh et al (2014) extensively reviewed previous studies and summarized that IF was effective in increasing antioxidant enzymes, in particular, glutathione activity (29). Furthermore, Arumugam et al (2010) showed that IF increased the HO-1 level in vulnerable brain regions, and the increased HO-1 level was correlated with decreasing infarct volume and neurological deficit following focal cerebral ischemic stroke in mice (26). Recently, Hu et al (2017) reported that postoperative IF for a week after chronic cerebral hypoperfusion in rats significantly decreased malondialdehyde (MDA) activity, maintained glutathione, SOD1, and SOD2 levels in the hippocampus, and improved memory deficit induced by the hypoperfusion (30). In our present study, we found that
SOD2 and CAT immunoreactivities were hardly shown in CA pyramidal cells in the IF+Ischemia group; instead, the immunoreactivities were increased in non-pyramidal cells, which were found to be astrocytes $(31,32)$, compared to the Ischemia group. Nevertheless, there was no IF-mediated neuronal protection in the hippocampal CA1 following tGCI. Our present study indicates that IF could increase SOD2 and CAT expressions in CA1 pyramidal cells in the IF+Sham groups and in non-pyramidal cells in the IF+Ischemia group but does not protect neurons from ischemic injury in a gerbil model of tGCI, which is different from transient focal cerebral ischemia.

Activations of microglia and astrocytes in the acute phase of post-ischemia are increased in response to ischemia-induced neuronal damage in the gerbil hippocampal CA1 $(18,33)$. The activations (gliosis) are one of the main reasons for the secondary damage that increases cytokine production during neuronal degeneration after ischemia $(34,35)$. Our previous studies have shown that the attenuation of glial activation is strongly correlated with the protection of hippocampal CA1 
A

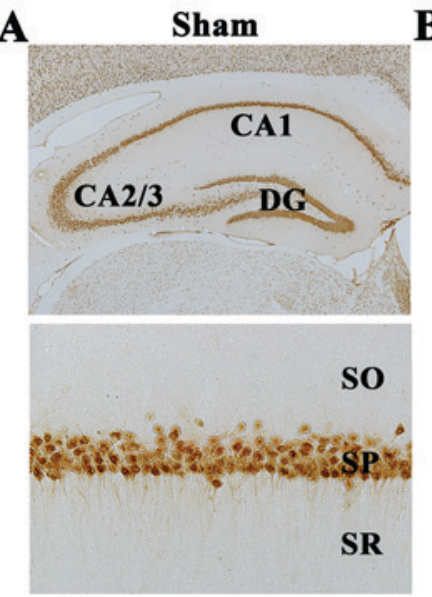

B

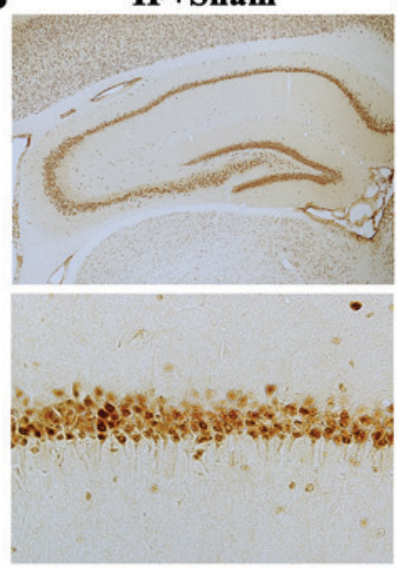

E

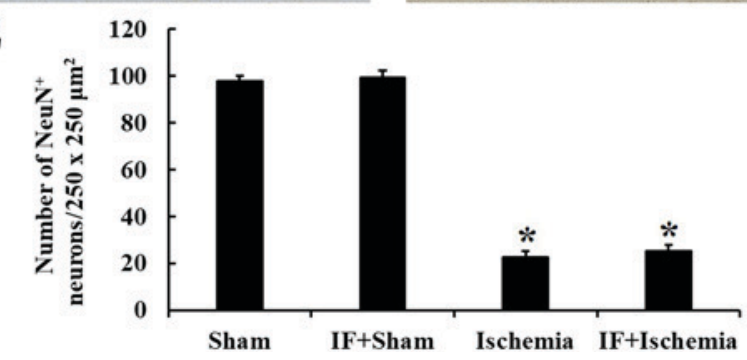

C
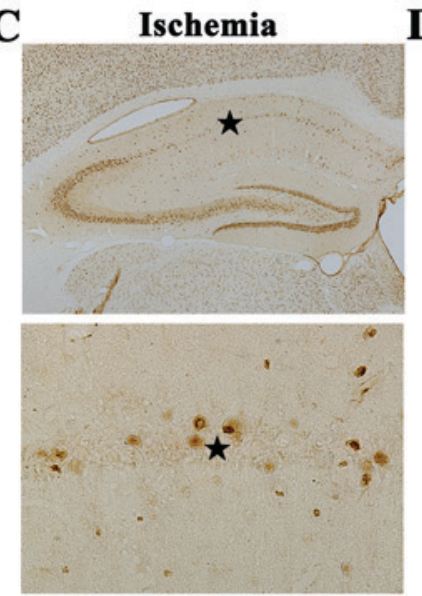

D
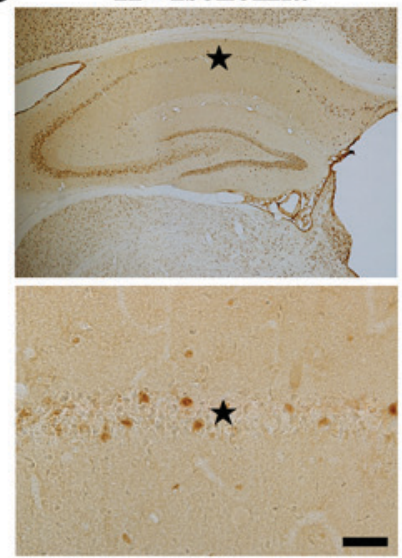

Figure 6. NeuN immunohistochemistry. NeuN immunoreactive neurons in the CA1 of (A) Sham, (B) IF+Sham, (C) Ischemia, and (D) IF+Ischemia groups 5 days after tGCI. A few NeuN immunoreactive pyramidal neurons are shown in the stratum pyramidale (SP, star in C) in the Ischemia group. In the IF+Ischemia group, numbers of NeuN-immunoreactive pyramidal neurons (star in D) are similar to the Ischemia group. The scale bar represents $400 \mu \mathrm{m}$ for the top row and $50 \mu \mathrm{m}$ for the bottom row. (E) Number of NeuN-immunoreactive neurons per $250 \times 250 \mu \mathrm{m}^{2}$ in the CA1 (n=7 in each group, ${ }^{*}<0.05 \mathrm{vs}$. Sham group). Bars indicate the means \pm SEM. CA, cornu ammonis; DG, dentate gyrus; SO, stratum oriens; SR, stratum radiatum.

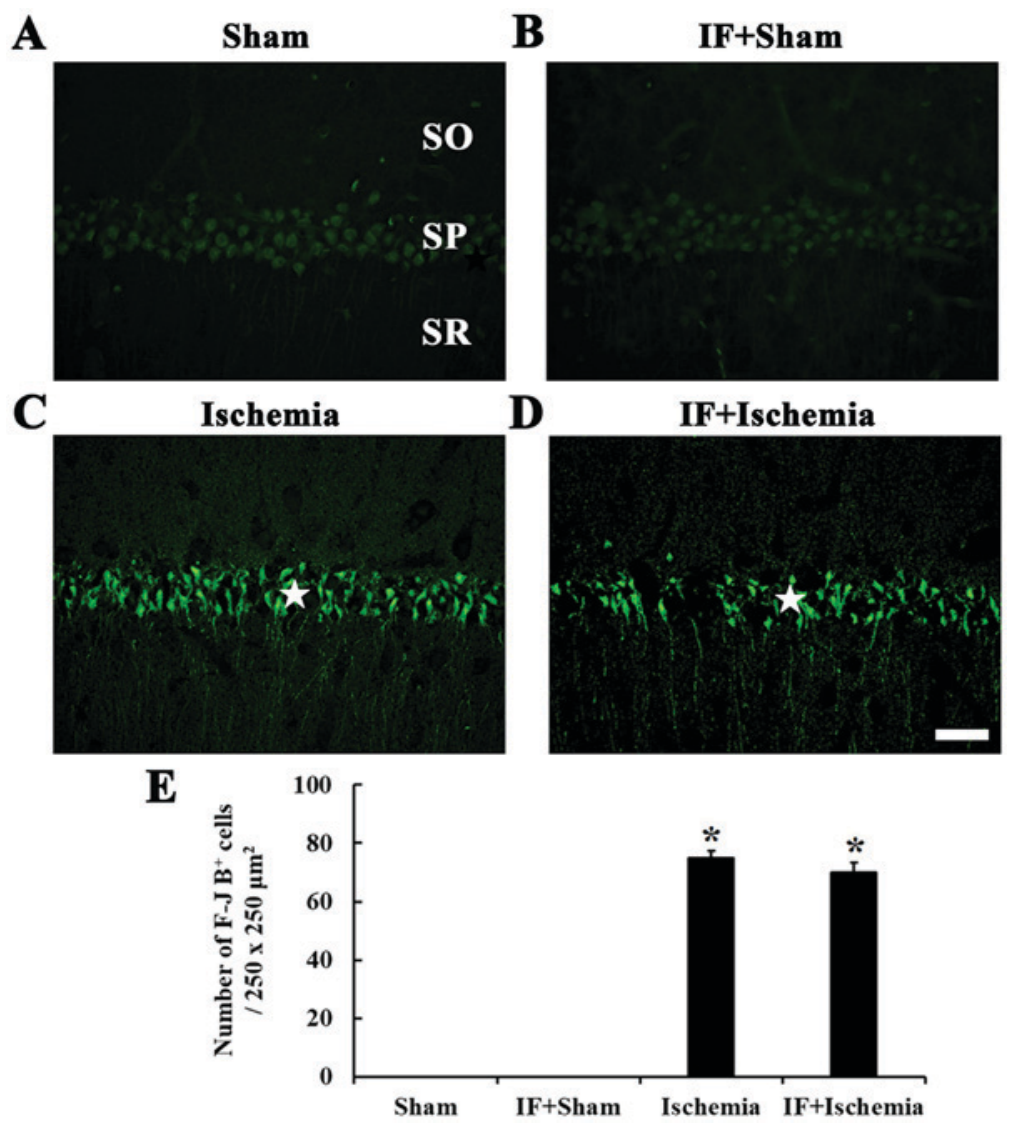

Figure 7. F-J B histofluorescence staining. F-J B positive cells in the CA1 of (A) Sham, (B) IF+Sham, (C) Ischemia, and (D) IF+Ischemia groups 5 days after tGCI. In the Ischemia group, many F-J B-positive cells (star in C) are detected in the stratum pyramidale (SP). In the IF+Ischemia group, numbers of F-J B-positive cells (star in D) are similar to the Ischemia group. Scale bar=50 $\mu \mathrm{m}$. (E) Number of F-J B positive cells per $250 x 250 \mu \mathrm{m}^{2}$ in the CA1 ( $\mathrm{n}=7$ in each group, ${ }^{*} \mathrm{P}<0.05$ vs. Sham group). Bars indicate the means \pm SEM. SO, stratum oriens; SR, stratum radiatum. 

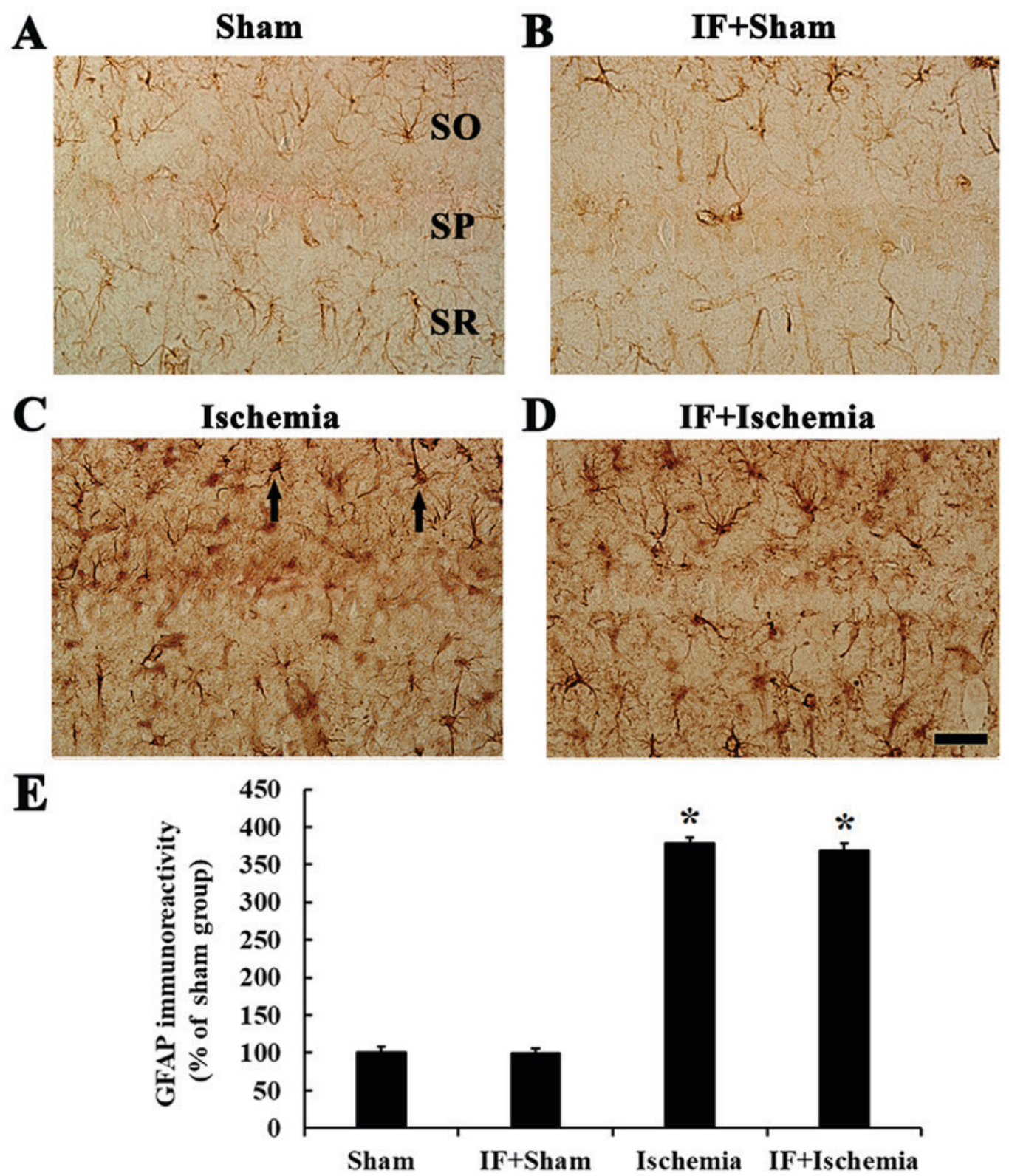

Figure 8. GFAP immunohistochemistry. (A-D) Immunoreactivities of GFAP in the CA1 of Sham, IF+Sham, Ischemia, and IF+Ischemia groups 5 days after tGCI. In the Ischemia group, GFAP-immunoreactive cells (arrows in C) are hypertrophied, and GFAP immunoreactivity is significantly increased compared to the Sham and IF+Sham groups. GFAP immunoreactivity in the IF+Ischemia group is not different from the Ischemia group. Scale bar=50 $\mu \mathrm{m}$. (E) GFAP immunoreactivity as percent values in the $\mathrm{CA} 1$ area $\left(\mathrm{n}=7\right.$ in each group, ${ }^{*} \mathrm{P}<0.05$ vs. Sham group). Bars indicate the means $\pm \mathrm{SEM}$. SO, stratum oriens; $\mathrm{SP}$, stratum pyramidale; SR, stratum radiatum.

neurons from $\mathrm{tGCI}(36,37)$. It was reported that three months of IF could decrease seizure-induced microgliosis in the lesioned hippocampus (38). It was reported that postoperative IF for a week after chronic cerebral hypoperfusion in rats significantly attenuated microglial activation in the hippocampus induced by chronic cerebral hypoperfusion (30). Although postoperative IF reduces injury-induced microglial activation in the hippocampus, as shown in our present study, preoperative IF prior to tGCI does not inhibit activations of microglia and astrocytes in the hippocampus induced by tGCI.

In summary, our results showed that preoperative IF increased immunoreactivities of SOD2 and CAT in CA1 pyramidal cells before tGCI and in non-pyramidal cells after tGCI. However, the IF did not protect death of CA1 pyramidal neurons following tGCI in gerbils. In this regard, we need to study the causes of the failure in protecting CA1 pyramidal neurons after 5 min of tGCI.

\section{Acknowledgements}

Not applicable.

\section{Funding}

The present study was supported by Basic Science Research Program through the National Research Foundation of Korea (NRF) funded by the Ministry of Education (grant no. NRF-2015R1D1A1A01059728), by the Bio \& Medical Technology Development Program of the NRF funded by the Korean government, MSIP (grant 
$\mathbf{A}$

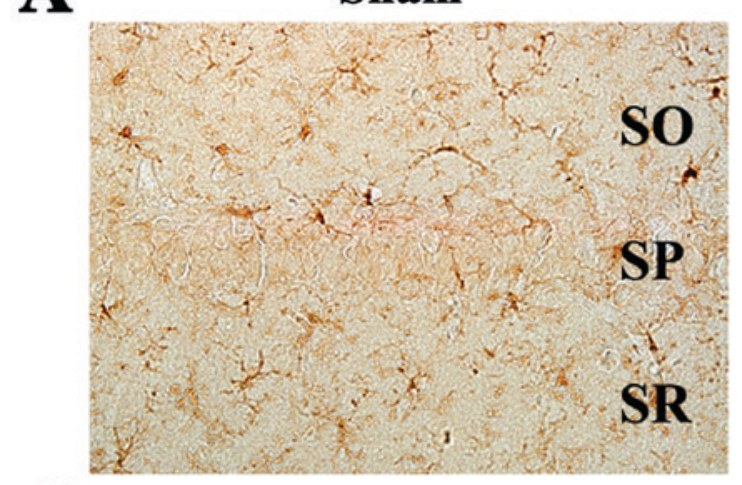

C
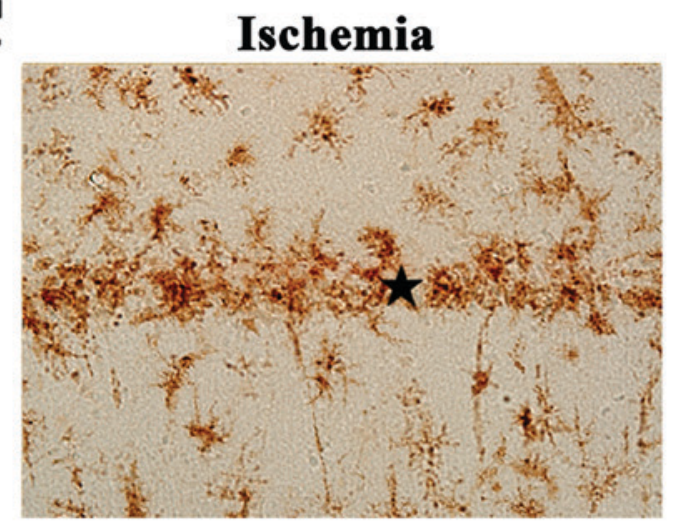

$\mathbf{E}$

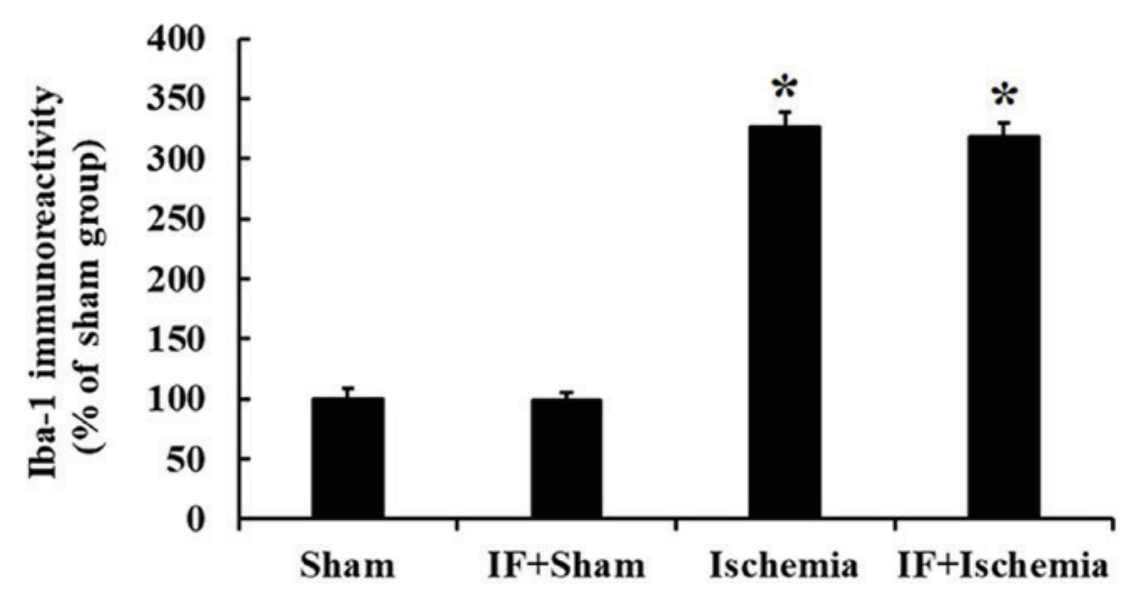

B

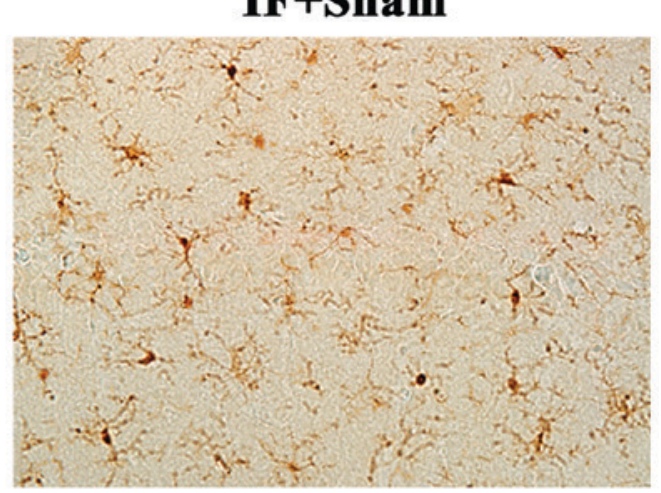

D

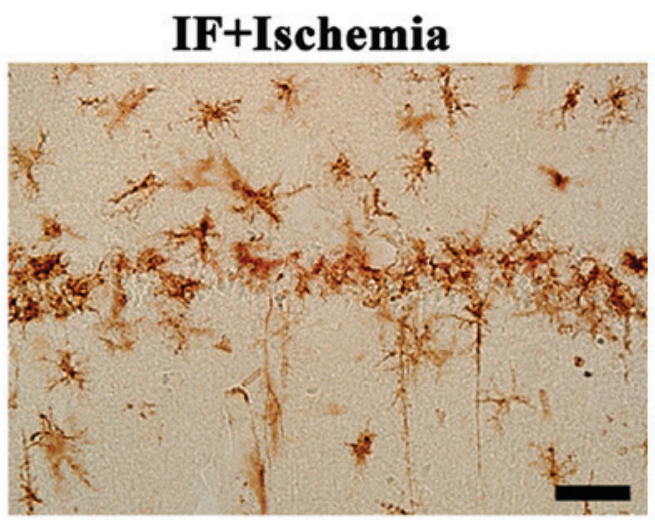

Figure 9. Iba-1 immunohistochemistry. (A-D) Immunoreactivities of Iba-1 in the CA1 of Sham, IF+Sham, Ischemia, and IF+Ischemia groups 5 days after tGCI. Iba-1-immunoreactive cells in the Ischemia group are aggregated in the stratum pyramidale (SP, star in C), and the immunoreactivity is significantly increased compared to the Sham and IF+Sham groups. Iba-1 immunoreactivity in the IF+Ischemia group is not different from the Ischemia group. Scale bar $=50 \mu \mathrm{m}$. (E) Iba-1 immunoreactivity as percent values in the CA1 area ( $\mathrm{n}=7$ in each group, ${ }^{*} \mathrm{P}<0.05$ vs. Sham group). Bars indicate the means $\pm \mathrm{SEM}$. SO, stratum oriens; SR, stratum radiatum.

no. NRF-2015M3A9B6066968), by Basic Science Research Program through the National Research Foundation of Korea (NRF) funded by the Ministry of Science, ICT \&Future Planning (grant no. NRF-2017R1A2B4009079), and by Korea Institute of Planning and Evaluation for Technology in Food, Agriculture, Forestry (IPET) through High Value-added Food Technology Development Program, funded by Ministry of Agriculture, Food and Rural Affairs (MAFRA) (grant no. 117055-3).

\section{Availability of data and materials}

All data generated or analyzed during the present study are included in this published article.

\section{Authors' contributions}

YN, BS, TL, MS and HK performed the measurements. SK, JP, JL, JY, IK and YL analyzed and interpreted data. JA, MW and JK made substantial contributions to conception and design, and were involved in drafting, revising the manuscript and interpreting all data. All authors read and approved the final manuscript.

\section{Ethics approval and consent to participate}

The protocol of this experiment was approved by the Institutional Animal Care and Use Committee (IACUC) at Kangwon National University (approval no. KW-180124-1). 


\section{Patient consent for publication}

Not applicable.

\section{Competing interests}

The authors declare that they have no competing interests.

\section{References}

1. Kirino T and Sano K: Selective vulnerability in the gerbil hippocampus following transient ischemia. Acta Neuropathol 62: 201-208, 1984

2. Hou ST and MacManus JP: Molecular mechanisms of cerebral ischemia-induced neuronal death. Int Rev Cytol 221: 93-148, 2002.

3. Mehta SL, Manhas N and Raghubir R: Molecular targets in cerebral ischemia for developing novel therapeutics. Brain Res Rev 54: 34-66, 2007.

4. Warner DS, Sheng H and Batinic-Haberle I: Oxidants, antioxidants and the ischemic brain. J Exp Biol 207: 3221-3231, 2004.

5. Gilgun-Sherki Y, Rosenbaum Z, Melamed E and Offen D: Antioxidant therapy in acute central nervous system injury: Current state. Pharmacol Rev 54: 271-284, 2002

6. Brown JE, Mosley M and Aldred S: Intermittent fasting: A dietary intervention for prevention of diabetes and cardiovascular disease? Br J Diabetes Vasc Dis 13: 68-72, 2013.

7. Kim J, Kang SW, Mallilankaraman K, Baik SH, Lim JC, Balaganapathy P, She DT, Lok KZ, Fann DY, Thambiayah U, et al: Transcriptome analysis reveals intermittent fasting-induced genetic changes in ischemic stroke. Hum Mol Genet 27: 1497-1513, 2018.

8. Mattson MP, Longo VD and Harvie M: Impact of intermittent fasting on health and disease processes. Ageing Res Rev 39: 46-58, 2017.

9. Tajes M, Gutierrez-Cuesta J, Folch J, Ortuño-Sahagun D, Verdaguer E, Jiménez A, bJunyent F, Lau A, Camins A and Pallàs M: Neuroprotective role of intermittent fasting in senescence-accelerated mice P8 (SAMP8). Exp Gerontol 45: 702-710, 2010.

10. Martin B, Mattson MP and Maudsley S: Caloric restriction and intermittent fasting: Two potential diets for successful brain aging. Ageing Res Rev 5: 332-353, 2006.

11. Zhu H, Guo Q and Mattson MP: Dietary restriction protects hippocampal neurons against the death-promoting action of a presenilin-1 mutation. Brain Res 842: 224-229, 1999.

12. Jeong JH, Yu KS, Bak DH, Lee JH1, Lee NS, Jeong YG, Kim DK, Kim JJ3 and Han SY: Intermittent fasting is neuroprotective in focal cerebral ischemia by minimizing autophagic flux disturbance and inhibiting apoptosis. Exp Ther Med 12: 3021-3028, 2016.

13. Fann DY, Santro T, Manzanero S, Widiapradja A, Cheng YL, Lee SY, Chunduri P, Jo DG, Stranahan AM, Mattson MP and Arumugam TV: Intermittent fasting attenuates inflammasome activity in ischemic stroke. Exp Neurol 257: 114-119, 2014

14. Yu ZF and Mattson MP: Dietary restriction and 2-deoxyglucose administration reduce focal ischemic brain damage and improve behavioral outcome: Evidence for a preconditioning mechanism. J Neurosci Res 57: 830-839, 1999.

15. Kirino T: Delayed neuronal death in the gerbil hippocampus following ischemia. Brain Res 239: 57-69, 1982.

16. Ginsberg MD and Busto R: Rodent models of cerebral ischemia Stroke 20: 1627-1642, 1989.

17. Li L, Wang Z and Zuo Z: Chronic intermittent fasting improves cognitive functions and brain structures in mice. PLoS One 8 : e66069, 2013.

18. Lee CH, Yoo KY, Choi JH, Park OK, Hwang IK, Kim SK, Kang IJ, Kim YM and Won MH: Neuronal damage is much delayed and microgliosis is more severe in the aged hippocampus induced by transient cerebral ischemia compared to the adult hippocampus. J Neurol Sci 294: 1-6, 2010.

19. Lee TK, Park JH, Ahn JH, Shin MC, Cho JH, Bae EJ, Kim YM, Won $\mathrm{MH}$ and Lee $\mathrm{CH}$ : Pretreated duloxetine protects hippocampal CA1 pyramidal neurons from ischemia-reperfusion injury through decreases of glial activation and oxidative stress. J Neurol Sci 370: 229-236, 2016
20. Candelario-Jalil E, Alvarez D, Merino N and León OS: Delayed treatment with nimesulide reduces measures of oxidative stress following global ischemic brain injury in gerbils. Neurosci Res 47: 245-253, 2003

21. Goodrick CL, Ingram DK, Reynolds MA, Freeman JR and Cider N: Effects of intermittent feeding upon body weight and lifespan in inbred mice: Interaction of genotype and age. Mech Ageing Dev 55: 69-87, 1990.

22. Armogida M, Nistico R and Mercuri NB: Therapeutic potential of targeting hydrogen peroxide metabolism in the treatment of brain ischaemia. Br J Pharmacol 166: 1211-1224, 2012.

23. Lebovitz RM, Zhang H, Vogel H, Cartwright J Jr, Dionne L, Lu N, Huang S and Matzuk MM: Neurodegeneration, myocardial injury, and perinatal death in mitochondrial superoxide dismutase-deficient mice. Proc Natl Acad Sci USA 93: 9782-9787, 1996.

24. Ho YS, Gargano M, Cao J, Bronson RT, Heimler I and Hutz RJ: Reduced fertility in female mice lacking copper-zinc superoxide dismutase. J Biol Chem 273: 7765-7769, 1998.

25. Dringen R, Pawlowski PG and Hirrlinger J: Peroxide detoxification by brain cells. J Neurosci Res 79: 157-165, 2005.

26. Arumugam TV, Phillips TM, Cheng A, Morrell CH, Mattson MP and Wan R: Age and energy intake interact to modify cell stress pathways and stroke outcome. Ann Neurol 67: 41-52, 2010

27. Yan BC, Park JH, Shin BN, Ahn JH, Kim IH, Lee JC, Yoo KY, Hwang IK, Choi JH, Park JH, et al: Neuroprotective effect of a new synthetic aspirin-decursinol adduct in experimental animal models of ischemic stroke. PLoS One 8: e74886, 2013.

28. Yan BC, Park JH, Ahn JH, Kim IH, Park OK, Lee JC, Yoo KY, Choi JH, Lee CH, Hwang IK, et al: Neuroprotection of posttreatment with risperidone, an atypical antipsychotic drug, in rat and gerbil models of ischemic stroke and the maintenance of antioxidants in a gerbil model of ischemic stroke. J Neurosci Res 92: 795-807, 2014

29. Walsh ME, Shi Y and Van Remmen H: The effects of dietary restriction on oxidative stress in rodents. Free Radic Biol Med 66: 88-99, 2014.

30. Hu Y, Zhang M, Chen Y, Yang Y and Zhang JJ: Postoperative intermittent fasting prevents hippocampal oxidative stress and memory deficits in a rat model of chronic cerebral hypoperfusion. Eur J Nutr, Jan 11, 2018 (Epub ahead of print).

31. Chen KY, Chiu CH and Wang LC: Anti-apoptotic effects of Sonic hedgehog signalling through oxidative stress reduction in astrocytes co-cultured with excretory-secretory products of larval Angiostrongylus cantonensis. Sci Rep 7: 41574, 2017.

32. Lee JC, Park JH, Kim IH, Cho GS, Ahn JH, Tae HJ, Choi SY, Cho JH, Kim DW, Kwon YG, et al: Neuroprotection of ischemic preconditioning is mediated by thioredoxin 2 in the hippocampal CA1 region following a subsequent transient cerebral ischemia. Brain Pathol 27: 276-291, 2017.

33. Yan BC, Park JH, Ahn JH, Choi JH, Yoo KY, Lee CH, Cho JH, Kim SK, Lee YL, Shin HC and Won MH: Comparison of glial activation in the hippocampal CA1 region between the young and adult gerbils after transient cerebral ischemia. Cell Mol Neurobiol 32: 1127-1138, 2012.

34. Patel AR, Ritzel R, McCullough LD and Liu F: Microglia and ischemic stroke: A double-edged sword. Int J Physiol Pathophysiol Pharmacol 5: 73-90, 2013.

35. Kawabori $M$ and Yenari MA: Inflammatory responses in brain ischemia. Curr Med Chem 22: 1258-1277, 2015.

36. Park JH, Park CW, Ahn JH, Choi SY, Shin MC, Cho JH, Lee TK, $\mathrm{Kim} \mathrm{IH}$, Cho JH, Lee JC, et al: Neuroprotection and reduced gliosis by pre- and post-treatments of hydroquinone in a gerbil model of transient cerebral ischemia. Chem Biol Interact 278: 230-238, 2017.

37. Park CW, Lee TK, Cho JH, Kim IH, Lee JC, Shin BN, Ahn JH, Kim SK, Shin MC, Ohk TG, et al: Rufinamide pretreatment attenuates ischemia-reperfusion injury in the gerbil hippocampus. Neurol Res 39: 941-952, 2017

38. Lee J, Auyeung WW and Mattson MP: Interactive effects of excitotoxic injury and dietary restriction on microgliosis and neurogenesis in the hippocampus of adult mice. Neuromolecular Med 4: 179-196, 2003.

This work is licensed under a Creative Commons Attribution-NonCommercial-NoDerivatives 4.0 International (CC BY-NC-ND 4.0) License. 